\title{
Urban rural differentials in spatial distribution of pregnant women having received Tetanus Toxoid injection by district in Punjab: Results from Pakistan Social and Living Standards Measurements Survey 2014-15
}

Masood Ali Shaikh

Dear Madam, Tetanus is a potentially fatal infection caused by Clostridium tetani bacteria, and during childbirth it can infect wounds and results in maternal death.1,2 Tetanus Toxoid (TT) vaccination is a simple, effective and freely available preventive measure available in public sector health facilities in Pakistan for pregnant women that can protect pregnant women and their newborns. Globally, TT vaccination is a standard preventive tool in the arsenal of primary health care service providers. In this study, district level TT vaccination coverage in pregnant women during their last pregnancy, in the Punjab province was mapped using Geographic information Systems to highlight its spatial distribution disaggregated by urban, rural residency status and the differences between the two types of dwellers.

The Pakistan Bureau of Statistics regularly conducts 'Pakistan Living Standards Measurement' surveys (PSLM). ${ }^{3}$ The latest freely available PSLM 2014-15 district-level survey data as a survey report in tabular form was downloaded from the Pakistan Bureau of Statistics website as PDF file. ${ }^{4}$ Punjab districts data was entered into a spreadsheet and joined with the Punjab districts shapefile in GIS programme ArcGIS 10.7. Three choropleth maps using ArcGIS 10.7, were created depicting percentage of pregnant women that received tetanus toxoid (TT) injection in urban, rural, and the differences between urban and rural residents by district. Four classes were used to depict various percentage levels of immunization status, using natural breaks (Jenks) method for the map for urban residency status. For rural residency status, again four classes were used, but with manually defined intervals to match the urban map for meaningful district-level comparisons. While for the differences between urban residency percentages compared to rural residency percentages, six manually defined classes were used to bring out more nuanced differentials.

Figure-1 shows the map of Punjab province showing all 36 districts along with their names. Figure- 2 shows three maps of TT immunization percentages by urban (left-side map), Independant Consultant, Karachi, Pakistan.

Correspondence: Masood Ali Shaikh e-mail: masoodalishaikh@gmail.com

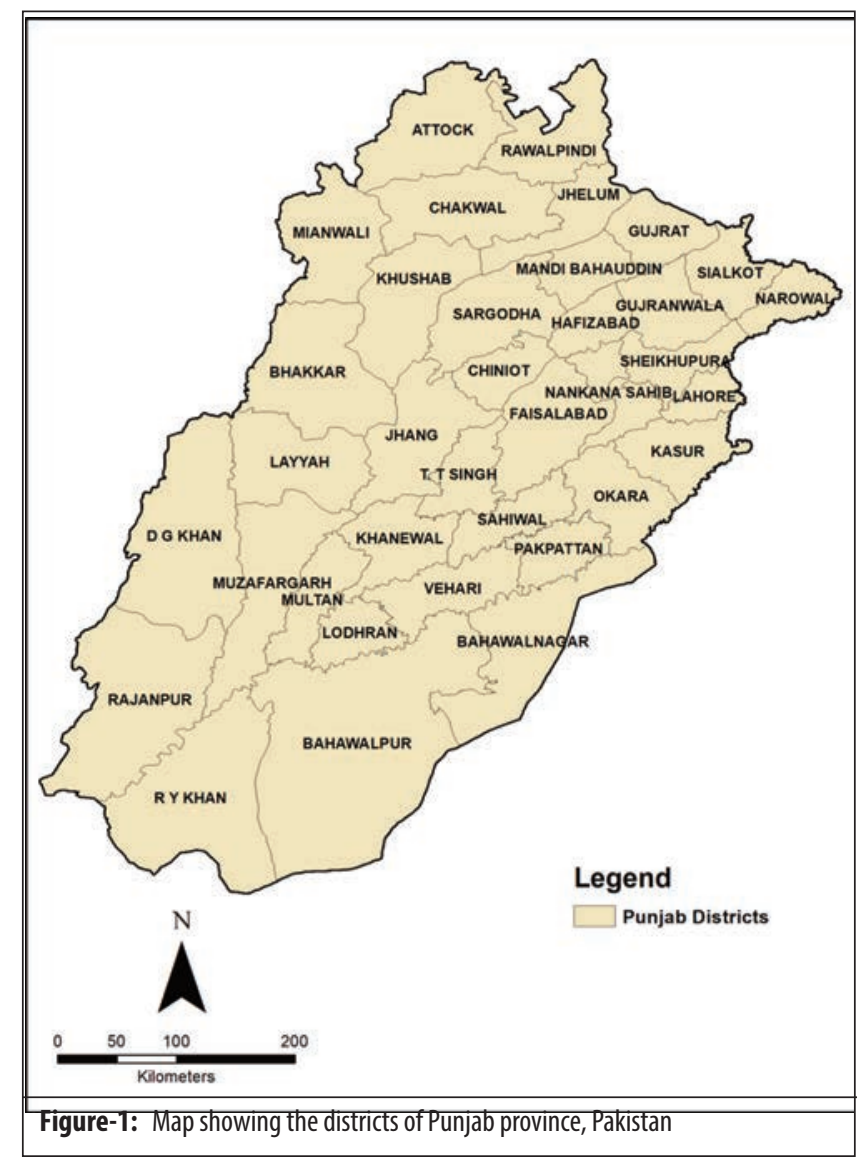

rural (central map) residency status, and the differences between the two (right-side map). The TT immunization percentages ranged from $56 \%$ to $100 \%$ among urban dwellers, with districts of Rahim Yar Khan and PakPattan falling in the lowest group of $56 \%-70 \%$. While in seven districts the percentages ranged from 95\%-100\%. Among rural dwellers, the TT immunization percentages ranged from $57 \%-70 \%$, with six districts falling in the lowest group of $57 \%-70 \%$ coverage. While in five districts the percentages ranged from $95 \%-98 \%$ coverage. The differences between the urban and rural TT immunization district percentages ranged from $-13 \%$ to $18 \%$; with negative differences between urban and rural residency status implying that rural residents in the district had higher percentages compared to urban residents. Kasur 


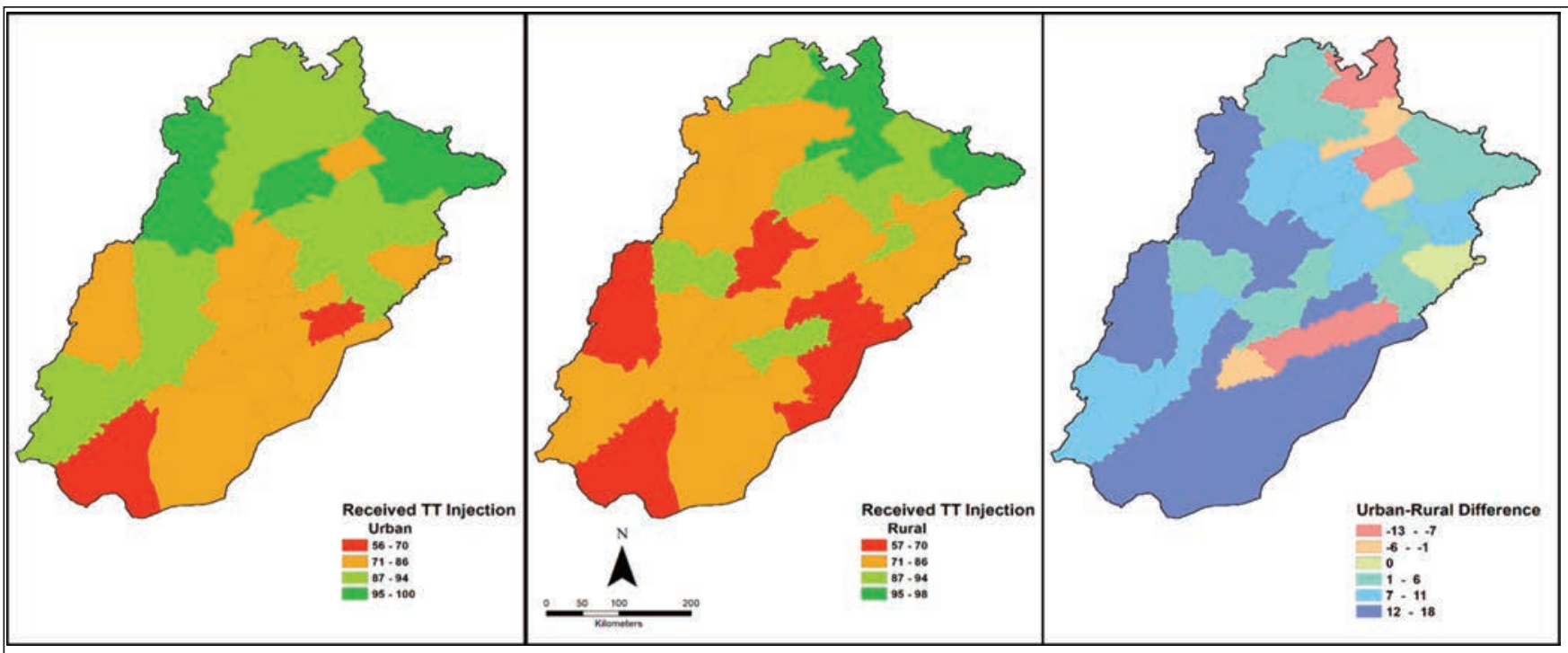

Figure-2: Map showing the percentages of Tetanus Toxoid vaccination for pregnant women during their last pregnancy by district in Punjab, disaggregated by urban, rural, and urban-rural differentials. Left map showing the percentages of Tetanus Toxoid vaccination for pregnant women during their last pregnancy by district in urban dwellers, middle map showing the percentages in rural residents, and the right map showing urban-rural differentials.

was the only district with no percentage difference among the urban and rural pregnant women receiving TT immunization. In seven districts rural residents had higher percentages compared to urban districts, with biggest difference of $-13 \%$ in PakPattan, and the lowest difference of $-1 \%$ in Jehlum. While in 28 districts urban residents had higher percentages compared to rural residents, with highest difference of $18 \%$ in Bahawalnager and lowest difference of $1 \%$ in Khanewal districts.

For both urban and rural dwellers, the lowest percentages were reported for the districts of Rahim Yar Khan and PakPattan. While in none of the rural districts the TT overage percentages were $100 \%$. Regarding immunization percentages in the urban areas, with the exception of Mandi Bahauddin, Kasur, and D.G. Khan districts, all districts in the north and western part of the province reported percent coverage ranging from $87 \%-100 \%$. For rural residents, the highest percentages were reported for the eleven northern districts in the range of $87 \%-98 \%$. The two districts from central part of the province i.e.Layyah and Vehari were the only exceptions, that also fell in this high range. In general, southern and eastern districts reported lower percentages for both urban and rural districts.

This is the first study on mapping of urban-rural residency disaggregated district level representative data on Tetanus
Toxoid immunization in the province of Punjab for pregnant women, during their last pregnancy. Several interesting patterns emerged when studying urban, rural, and the differential maps. The PSLM district level data for the 2014-15 survey is albeit dated, but it provides baseline profile of TT percentage coverage to facilitate district level immunization trends analysis with future PSLMs.

Disclaimer: None.

Conflicts of interest: None.

Funding disclosure: None.

\section{DOI: https://doi.org/10.47391/JPMA.22-008}

\section{References}

1. Abu-Raya B, Maertens K, Edwards KM, Omer SB, Englund JA, Flanagan $\mathrm{KL}$, et al. Global Perspectives on Immunization During Pregnancy and Priorities for Future Research and Development: An International Consensus Statement. Front Immunol. 2020;11:1282.

2. Abu-Raya B, Maertens K. Protection of the Newborn Through Vaccination in Pregnancy. Neoreviews. 2021;22:e25-e39.

3. Pakistan Bureau of Statistics. 'Pakistan Social And Living Standards Measurement Survey (PSLM) 2014-15 Provincial / District' report. (Accessed: Feb13, 2019)

4. Pakistan Bureau of Statistics. Pakistan Social and Living Standards Measurement. http://www.pbs.gov.pk/content/pakistan-social-andliving-standards-measurement (Accessed: Feb 13, 2019). 